\title{
Brooding behaviour and cost of brooding in small body size brachyuran crabs
}

\author{
Miriam Fernández ${ }^{1,2, *}$, Ricardo Calderón ${ }^{1}$, Mauricio Cifuentes ${ }^{1,3}$, Paula Pappalardo ${ }^{1}$ \\ ${ }^{1}$ Estación Costera de Investigaciones Marinas and Center for Advanced Studies in Ecology and Biodiversity, Departamento \\ de Ecología, Facultad de Ciencias Biológicas, Pontificia Universidad Católica, Alameda 340, Casilla 114-D, Santiago, Chile \\ ${ }^{2}$ LIA DIAMS, Evolution et Génetique des Populations Marines, AD2M UMR 7144 CNRS-UPMC, LIA DIAMS, \\ Station Biologique, Place Georges Teisser, BP74, 29682 Roscoff cedex, France \\ ${ }^{3}$ Present address: Facultad de Ciencias del Mar, Universidad Católica del Norte, Casilla 117, Coquimbo, Chile
}

\begin{abstract}
Constraints in the capacity to brood, specifically supplying oxygen to aggregated embryo masses, is one of the mechanisms invoked to explain the association between small body size and brooding exhibited by marine invertebrates. We hypothesized that brooding costs may affect the capacity to supply oxygen to the brood, therefore constraining brooding to small body size. We analyzed female brooding behaviour and patterns of oxygen availability in embryo masses of females carrying early and late stage embryos in 2 species of small size crabs: Pisoides edwardsi and Acanthocyclus gayi. These species are between 3 and 10 times smaller than previously studied crab species. The costs of incubation were estimated by comparing oxygen consumption of nonbrooding and brooding females in each crab species. The same brooding behaviours reported for large body size species were performed by the 2 small species studied. However, behavioural patterns throughout embryo development were different between small and large body size species. Oxygen availability (\% air saturation) never showed limitations in embryo aggregations in the 2 small crab species, in contrast to results from previous studies on large crabs. Another clear difference between brooders with small and large body size is the cost of oxygen provision, as no differences in oxygen consumption of brooding and nonbrooding females were found in the 2 small species. In contrast, oxygen consumption of brooding females is twice that of nonbrooding females in crabs of large body size. Our results provide direct evidence for the effects of adult size on the costs of providing oxygen to the brood, suggesting that brooding at large body sizes may not be related to the capacity to hold a brood, but to the capacity to provide oxygen to the embryos, and to the associated cost. Further, we discuss the effect of embryo size on female brooding behaviour and brooding costs.
\end{abstract}

KEY WORDS: Body size - Brooding - Crab embryo - Decapods - Marine invertebrates · Oxygen provision $\cdot$ Parental care

Resale or republication not permitted without written consent of the publisher

\section{INTRODUCTION}

Brooding in marine animals is influenced by the physical characteristics of the environment (low solubility of oxygen, low diffusion coefficient), which affect oxygen acquisition in embryo aggregations (Strathmann \& Strathmann 1989, Fernández \& Brante 2003, Fernández et al. 2003, Green \& McCormick 2005). The low oxygen availability found in embryo aggregations of different types (gels, capsules, crab embryo masses; Cohen \& Strathmann 1996, Fernández et al. 2000,
Lardies \& Fernández 2002) negatively affect embryo development rate, hatching size and larval survival, particularly in large aggregations (Booth 1995, Strathmann \& Strathmann 1995, Cohen \& Strathmann 1996, Lee \& Strathmann 1998, Woods 1999, Lardies \& Fernández 2002, Fernández et al. 2003). Constraints in the capacity to brood, specifically supplying oxygen to aggregated embryo masses, is one of the mechanisms invoked to explain the association between small body size and brooding exhibited by marine invertebrates (Strathmann \& Strathmann 1982). It is remarkable that 
the same constraint to brood at large body size has been found in amphibians, a group that largely depends on aquatic systems for reproduction (Crump 1996). Moreover, embryo aggregations in amphibians also exhibit strong oxygen gradients (Seymour \& Roberts 1991, Seymour et al. 1991).

The evidence of the constraints to supply oxygen to embryo aggregations among marine invertebrates is mostly based on data on mollusks, polychaetes (Cohen \& Strathmann 1996), and decapod crustacean species (Fernández et al. 2002, 2003). However, these groups differ in the mechanisms used to provide oxygen. While polychaetes and gastropods employ passive mechanisms to aid oxygen diffusion to aggregated embryos, decapods exhibit active brooding behaviour directed towards ventilating embryo masses (Fernández et al. $2000,2002)$. Several studies have shown that structures produced by passively brooding species (e.g. gel) help oxygen diffusion to aggregated embryos (Strathmann \& Chaffee 1984). Among gastropods, gel investment per embryo increases allometrically with the thickness of egg masses (Lee \& Strathmann 1998), suggesting that the cost of oxygen provision among passive brooders is greater for large body size species. Thus, the limitations on incubation for large species may not only depend on the capacity to provide oxygen to the embryos, but also on the costs associated with providing oxygen. However, this is the only evidence supporting the links between (1) body size and physiological constraints on brooding, and (2) the capacity to provide oxygen and the costs associated with providing oxygen.

Marine crustaceans commonly exhibit active brooding behaviour directed towards ventilating the embryo masses, and within this group brooding is not constrained to small body sizes (Strathmann \& Strathmann 1982). Crustaceans always carry the embryos, regardless of their body size (e.g. peracarids in the thorax, decapods under the abdomen ), offering a good model to explore the relationship between body size and the cost of oxygen provision, which could shed light on the mechanisms determining the association between small body size and brooding found in aquatic animals. Decapod crustaceans are of particular interest because they exhibit a wide range of body sizes, including the largest marine brooders in nature. Besides, decapod crustaceans exhibit active brooding behaviour directed toward providing oxygen to developing embryos (Fernández et al. 2000, 2003). Decapod crustaceans are also interesting, because females are able to assess the oxygen demands of the embryos and change brooding behaviour according to the embryos' oxygen consumption (Baeza \& Fernández 2002, Fernández et al. 2003). This suggests that the oxygen requirements of the brood are an important evolutionary force influencing the incubatory behaviour of females in this group. The perfectly adjusted brooding behaviour of female crabs in response to oxygen requirements of the embryos (Baeza \& Fernández 2002, Fernández et al. 2003) also suggests that the costs of brooding are high. In fact, a 2 to 3 -fold increase in oxygen demand by brooding females has been reported for several species of Brachyura (Taylor \& Leelapiyanart 2001, Fernández et al. 2002, Baeza \& Fernández 2002, Brante et al. 2003, Fernández \& Brante 2003). Moreover, the high cost of brooding seems to affect investment in gonads and to determine the trade-off between the cost of brooding and the capacity to produce eggs (Brante et al. 2003). However, most of the studies that show high costs of active oxygen provisioning have been conducted on large brachyuran species, with carapace widths ranging between 80 and $170 \mathrm{~mm}$ and embryo masses between 6 and $10 \mathrm{~cm}$ diameter (Fernández et al. 2000, 2002, 2003, Baeza \& Fernández 2002, Brante et al. 2003, Fernández \& Brante 2003). We analyzed female brooding behaviour and patterns of oxygen availability in embryo masses of females carrying early and late stage embryos in 2 species of crabs of small size: Pisoides edwardsi and Acanthocyclus gayi. These species are between 3 and 10 times smaller than the previously studied crab species. The costs of incubation were estimated by comparing oxygen consumption of nonbrooding and brooding females for each crab species. We discuss our results in the context of previous findings on large bodied species and propose the cost of brooding as the possible mechanism behind the association between small body size and brooding reported for marine invertebrates (Strathmann \& Strathmann 1982).

\section{MATERIALS AND METHODS}

Collection and maintenance of study organisms. The 2 model species, Pisoides edwardsi and Acanthocyclus gayi, were selected because they are small and easily found. Females were collected in the intertidal zone of Las Cruces, Chile $\left(33^{\circ} 29^{\prime} \mathrm{S}, 71^{\circ} 38^{\prime} \mathrm{W}\right)$. A. gayi inhabits crevices, mussel beds and holdfasts of the seaweed Lessonia nigrescens. P. edwardsi is commonly found in tidal pools containing boulders covered by Gelidium chilensis, colonial bryozoans and sponges. Female crabs were transported to the laboratory at the Estación Costera de Investigaciones Marinas (Las Cruces) and maintained at constant temperature $\left(11\right.$ to $\left.12^{\circ} \mathrm{C}\right)$ in plastic aquaria $(\sim 0.5 \mathrm{l})$ with aerated seawater. Crabs were held under these conditions for acclimatization for at least $48 \mathrm{~h}$ before the experiments started. Brooding females were classified according to the developmental stage of the embryos in 2 broad categories: (1) early 
stage embryos: yolk was uniformly distributed, filling at least $75 \%$ of the entire embryo volume, and (2) late stage embryos: from initial pigmentation and visible eyes to well-developed eyes, pumping heart and visible appendages. Carapace width of females used in this study ranged between 10.4 and $14.3 \mathrm{~mm}$ for $P$. edwardsi (mean $=11.7 \mathrm{~mm}, \mathrm{SD}=0.9$ ) and 16.5 and $32.9 \mathrm{~mm}$ for A. gayi $($ mean $=21.2 \mathrm{~mm}, \mathrm{SD}=2.1)$.

Pattern of oxygen provision to the embryo mass. In order to evaluate whether the pattern of oxygen availability (\% air saturation) in the embryo mass varies throughout development, laboratory experiments were conducted following the experimental protocols proposed by Baeza \& Fernández (2002). Changes in oxygen availability in the center of the embryo mass in females of each species were monitored with a fiber optic oxygen sensor (Microptode Presens; Fernández et al. 2000). After calibration at 0 and $100 \%$ air saturation (using seawater saturated with $\mathrm{NaSO}_{3}$ and air, respectively), the fiber optic microptode was inserted into the center of the embryo mass (through a $1 \mathrm{~mm}$ diameter hole drilled in the female's third abdominal segment) and glued to the abdomen of the female. All experiments began $1 \mathrm{~h}$ after insertion of the microptode in order to allow the females to recover after manipulation. Previous studies had shown that the insertion of the microptode has no effect on the behaviour of other brooding brachyuran females (Baeza \& Fernández 2002). However, since we focused on 2 species considerably smaller than those previously studied, the effect of the microptode on female behaviour was evaluated (see below). Oxygen availability within the embryo mass was measured every $5 \mathrm{sec}$ for $2 \mathrm{~h}$. The experiments were conducted mostly during the day, although female behaviour and oxygen supply patterns do not show daily cycles (Ruiz-Tagle et al. 2002). For each species, the mean percent of time that the embryos were exposed to low ( $<25 \%$ air saturation) or high ( $>50 \%$ air saturation) oxygen availability was estimated. These limits were selected because: (1) oxygen conditions $<25 \%$ air saturation affect oxygen consumption of the embryos, and (2) oxygen conditions $>50 \%$ air saturation never influence oxygen consumption of the embryos (Naylor et al. 1999, Fernández et al. 2002). We conducted 7 replicates using females of Pisoides edwardsi carrying early stage embryos and 5 for females carrying late stage embryos. For Acanthocyclus gayi, 5 and 4 replicates were run for females carrying early and late stage embryos, respectively. A $t$-test for independent samples was used to compare the mean proportion of time that embryos were exposed to low $(<25 \%)$ or high $(>50 \%)$ air saturation between females carrying early and late stage embryos, for each species. The data were not transformed. The rationale to include 2 species was to eval- uate the consistency of patterns of oxygen availability, brooding behaviour and costs between females carrying early and late stage embryos in small body size crab species. Therefore, no comparison between species was conducted.

Behaviour of brooding females. The behaviour of brooding females was recorded using a Sony video recorder (Time lapse-Recorder, model SVT-3050) and a Sony vigilance video camera. For each replicate, a $1 \mathrm{~h}$ block was randomly selected to register female behaviour, considering sections of the video recordings in which females faced the camera because only observations at this angle allowed a reliable quantification of female behaviour. The following behaviours typical for brooding females (Baeza \& Fernández 2002) were registered: (1) abdominal flapping (quantified as number of events per hour); (2) manipulation of the embryo mass with the chelae (chelae poking, quantified as the proportion of time spent engaged in this activity); and (3) manipulation of the embryo mass using the pereiopods (pereiopod poking, quantified as the proportion of time devoted to this activity). The later behaviour was not exhibited by Pisoides edwardsi. Abdominal flapping has been associated with increases in oxygen availability in the center of the embryo masses of brachyuran crabs while chelae and pereiopod poking have been interpreted as behaviours allowing the assessment of oxygen conditions in the embryo mass (Cancridae: Baeza \& Fernández 2002; Portunidae: Fernández \& Brante 2003). Since nonbrooding females do not perform these behaviours (e.g. Baeza \& Fernández 2002), only brooding females were videotaped; 5 replicates for each embryo developmental stage were conducted for Acanthocyclus gayi, 7 for females of $P$. edwardsi carrying early stage embryos, and 8 for $P$. edwardsi carrying late stage embryos.

Although the microptode does not alter brooding behaviour of brachyuran crabs (Baeza \& Fernández 2002), the effect of the fiber optic on female behaviour was evaluated in Pisoides edwardsi. We selected this species as a model because it is 10 times smaller than previously studied crabs, and the smaller of the 2 species in this study (Baeza \& Fernández 2002, Fernández et al. 2003). A control group (without fiber optic) of brooding females was included in the experiment ( $\mathrm{n}=5$ for each stage). Independent 2-way ANOVAs (fixed factor) were conducted to compare each behaviour between females carrying embryos in early and late developmental stages, and between manipulation treatments (with and without fiber optic). Flapping frequency (number of events per hour) and percent of the experimental time that females were manipulating the embryo mass using the chelae were estimated. The latter variable was transformed to meet the assumption 
of the models (log transformation). For Acanthocyclus gayi, $t$-tests were conducted to compare each behaviour independently between females carrying embryos in early and late developmental stages. The proportion of time that the embryo mass was manipulated by the pereiopods was transformed (ln). No comparisons between species were conducted (see above).

Oxygen consumption of crab embryos and females. Oxygen consumption of crab embryos was measured using a double-wall closed microchamber $(2 \mathrm{ml})$, filled with $0.45 \mu \mathrm{m}$ filtered seawater. After calibration at $0 \%$ and $100 \%$ air saturation (using seawater saturated with $\mathrm{NaSO}_{3}$ and air, respectively), a small embryo sample (up to $0.1 \mathrm{~g}$ ) was placed into the microchamber, on a grid to avoid contact between the embryos and the stirring magnet (Naylor et al. 1999). The magnet was used to keep oxygen tension homogeneous in the microchamber. Oxygen consumption of the embryos was monitored with electrodes (Eschweiler); 8 replicates for each embryo stage were conducted for Pisoides edwardsi and 5 per embryo stage for Acanthocyclus gayi. For each species, mean oxygen consumption per $g$ wet weight was compared between embryos in early and late developmental stages using a $t$-test for independent samples. Data were transformed to meet model assumptions (ln for P. edwardsi, 1/x for A. gayi).

The experimental protocol proposed by Baeza \& Fernández (2002) was utilized to estimate the metabolic costs of brooding. Differences in oxygen consumption between brooding and nonbrooding females were used as a proxy of investment in brooding. Before the experiments started, calibration of the instruments was carried out at 0 and $100 \%$ air saturation as previously described. Oxygen consumption of females was measured using closed circuit respirometry (respiration chamber). The chamber volume was $70 \mathrm{ml}$ for Pisoides edwardsi and $153 \mathrm{ml}$ for Acanthocyclus gayi. Acclimated females were individually placed in the chamber filled with filtered seawater saturated with oxygen $(100 \%)$. The chambers were sealed and placed into a constant temperature bath $\left(80 \mathrm{l}_{;} 67 \times 40 \times 30 \mathrm{~cm}\right)$. Constant water flow from the chamber to the electrodes that registered oxygen depletion was maintained using a peristaltic pump. At least 5 replicates of each female condition and species were conducted (P. edwardsi; early embryos $=7$, late embryos $=9$, nonbrooding $=8 ; A$. gayi; early embryos $=5$, late embryos $=8$, nonbrooding $=5$ ). For brooding females, oxygen consumption per $g$ wet weight per minute was calculated by subtracting the estimated oxygen consumption of the embryo mass from the total oxygen consumption of females with the embryo mass. Oxygen consumption of the embryo mass was estimated taking into account the weight of the mass and the average oxygen consumption of the embryos (see above). A 1-way ANOVA was conducted to compare oxygen consumption between the 3 female conditions within species. Data were transformed to meet model assumptions (square-root transformation for P. edwardsi, ln for A. gayi).

\section{RESULTS}

\section{Patterns of oxygen provision to the embryo mass}

There were no significant differences in the mean percent of time that embryos were exposed to low oxygen availability $(<25 \%$ air saturation) between early and late developmental stages in Pisoides edwardsi $(t=0.889, \mathrm{df}=10, \mathrm{p}=0.395$, Fig. $1 \mathrm{~A})$ and Acanthocyclus gayi $(t=-1.39, \mathrm{df}=7, \mathrm{p}=0.206$, Fig. $1 \mathrm{D})$. Similarly, no significant differences were detected in the mean percent of time that early and late stage embryos were exposed at $>50 \%$ air saturation in both species (P. edwardsi, $t=0.52, \mathrm{df}=10, \mathrm{p}=0.61$ Fig. 1A; A. gayi, $t=1.57, \mathrm{df}=7, \mathrm{p}=0.159$; Fig. 1D). On average, embryos were exposed $5 \%$ of the time to $<25 \%$ air saturation and $77 \%$ of the time to oxygen conditions $>50 \%$ air saturation (Fig. 1A,D).

\section{Behaviour of brooding females}

Experimental manipulation and embryo developmental stage did not affect the average frequency, nor the duration of the brooding behaviours observed in Pisoides edwardsi (Table 1). No significant differences were found in the mean percent of time that females manipulated the embryo mass using the chelae between treatments (Table 1; Fig. 1B). Similarly, the mean frequency of abdominal flapping of females of $P$. edwardsi was not significantly different between manipulation treatments, nor between females incubating embryos in early and late development stages (Table 1; Fig. 1C).

The only behaviour showing similar patterns between the 2 study species was chelae poking, as no significant differences were found in the mean percent time that female Acanthocyclus gayi manipulated the embryo mass with their chelae between stage embryos $(t=0.82, \mathrm{df}=8, \mathrm{p}=0.43$; Fig. 1E). In contrast, the remaining brooding behaviour showed differences between embryo stages in $A$. gayi females (Fig. 1F). Females carrying late stage embryos showed a higher mean frequency of abdominal flapping $(t=3.42, \mathrm{df}=8$, $\mathrm{p}=0.009$; Fig. $1 \mathrm{~F})$ and a higher mean proportion of time devoted to manipulate the embryo mass using the pereiopods ( $t=2.79$, df $=8, \mathrm{p}=0.02$; Fig. $1 \mathrm{E}$ ) than females carrying early stage embryos. 

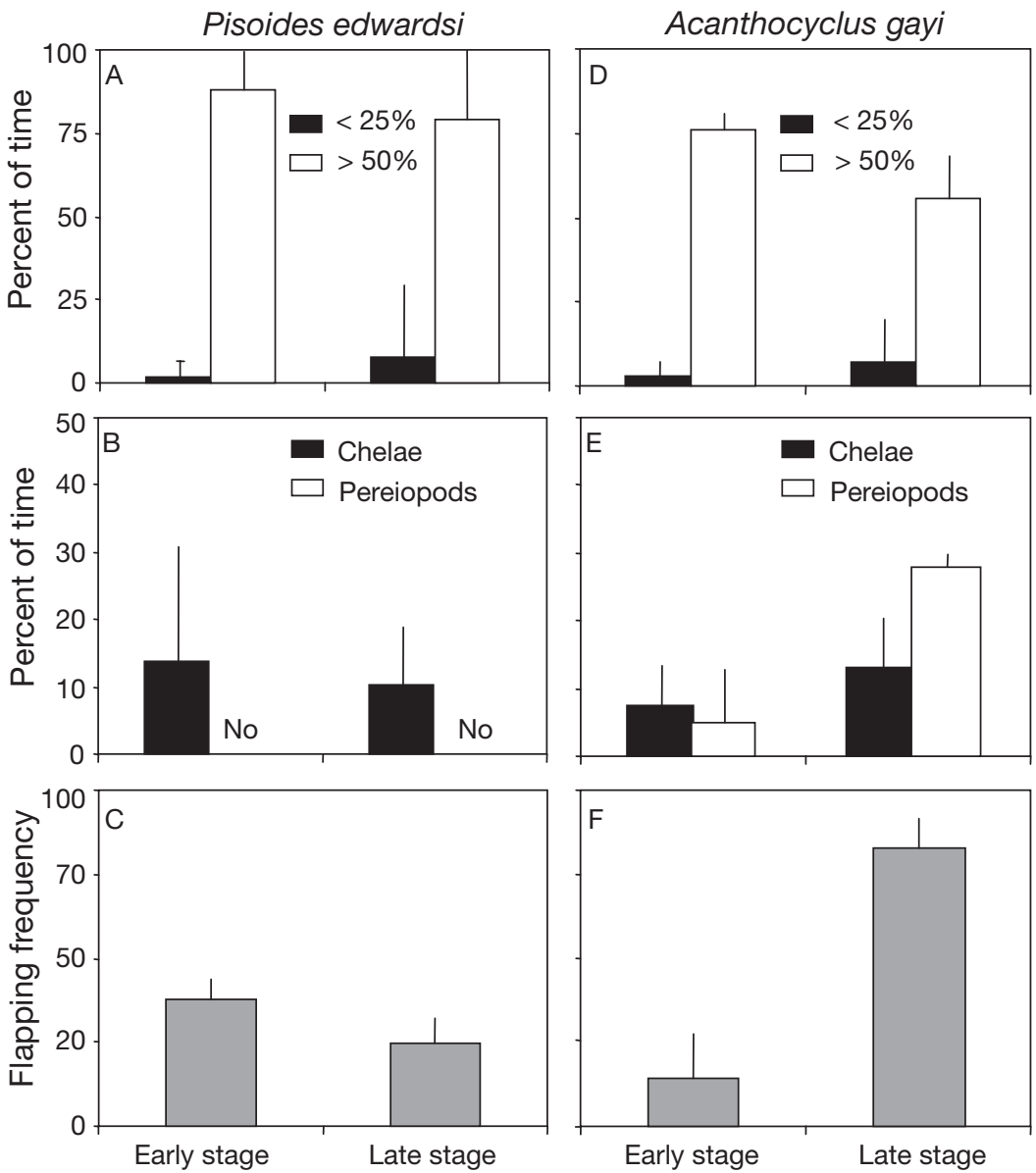

Fig. 1. Pisoides edwardsi and Acanthocylus gayi. (A,D) Time that embryos were exposed to low $(<25 \%)$ and high $(>50 \%)$ oxygen availability in the center of the embryo mass of females carrying early and late stage embryos. $(\mathrm{B}, \mathrm{E})$ Time that females devoted to manipulating the embryo mass using the chelae or pereiopods when carrying early and late stage embryos. (C,F) Frequency of abdominal flapping (number of events per hour) in females carrying early and late stage embryos. 'No' indicates that the behavior was not observed. Data are mean $+\mathrm{SD}$

Table 1. Pisoides edwardsi. Results of 2-way ANOVA conducted to compare the brooding behaviour between females carrying embryos in early and late developmental stages, considering the effect of the fiber optic microptode (manipulation treatment) on brooding behaviour

\begin{tabular}{|lrrrr|}
\hline Source of variation & SS & df & $F$ & $\mathrm{p}$ \\
\hline Abdominal flapping & & & & \\
$\quad$ Manipulation treatment & 6.29 & 1 & 0.03 & 0.88 \\
Embryo developmental stage & 128.12 & 1 & 0.47 & 0.50 \\
$\quad$ Treatment $\times$ Developmental stage & 1111.86 & 1 & 4.05 & 0.06 \\
Error & 5757.9 & 21 & & \\
Chelae poking & & & & \\
Manipulation treatment & 10.99 & 1 & 2.12 & 0.16 \\
Embryo developmental stage & 0.02 & 1 & 0.00 & 0.96 \\
Treatment $\times$ Developmental stage & 7.02 & 1 & 1.35 & 0.26 \\
Error & 10.04 & 21 & & \\
\hline
\end{tabular}

\section{Oxygen consumption of crab embryos and females}

Mean oxygen consumption of Pisoides edwardsi embryos was not influenced by embryo developmental stage ( $t$-test, $t=1.435, \mathrm{df}=14, \mathrm{p}=0.173$; Fig. 2A). A similar pattern was found for Acanthocyclus gayi ( $t$-test, $t=-1.54$, df $=8, \mathrm{p}=$ 0.16; Fig. 2A). Mean oxygen consumption was not significantly different between nonbrooding females and females carrying early or late stage embryos in, P. edwardsi $\left(F_{1,21}=1.21, \mathrm{p}=0.34\right)$ and $A$. $\operatorname{gayi}\left(F_{1,15}=1.27, \mathrm{p}=0.31\right.$; Fig. $\left.2 \mathrm{~B}\right)$.

\section{DISCUSSION}

Among brachyuran crabs, brooding females show behaviours that are not exhibited by nonbrooding females, such as abdominal flapping and manipulation of the embryo mass using the chelae and/or pereiopods (Cancridae: Baeza \& Fernández 2002; Portunidae: Fernández $\&$ Brante 2003). These behaviours have been observed in all the species analyzed to date (Maja squinado: Fernández et al. 2000; Cancer setosus: Baeza \& Fernández 2002; Homalaspis plana: Fernández et al. 2003; Ovalipes trimaculatus: Fernández \& Brante 2003), including Pisoides edwardsi and Acanthocylus gayi. However, the frequency at which these behaviours are performed seems to be related to adult size, although opposing trends in relation to body size seem to occur between behaviours that supply oxygen to the embryo mass and those that seem to assess oxygen conditions (Fig. 3A,B). The proportion of time that females devote to manipulate the embryo mass using the chelae, behaviour that has been related to the assessment of oxygen conditions in the embryo mass, seems to decrease as body size increases (Cancridae: Baeza \& Fernández 2002; Portunidae: Fernández \& Brante 2003; Fig. 3A). However, no differences between the 2 species included in our study were detected, even though the mean size of A. gayi is twice the size of $P$. edwardsi. This suggests that the effect of body size 


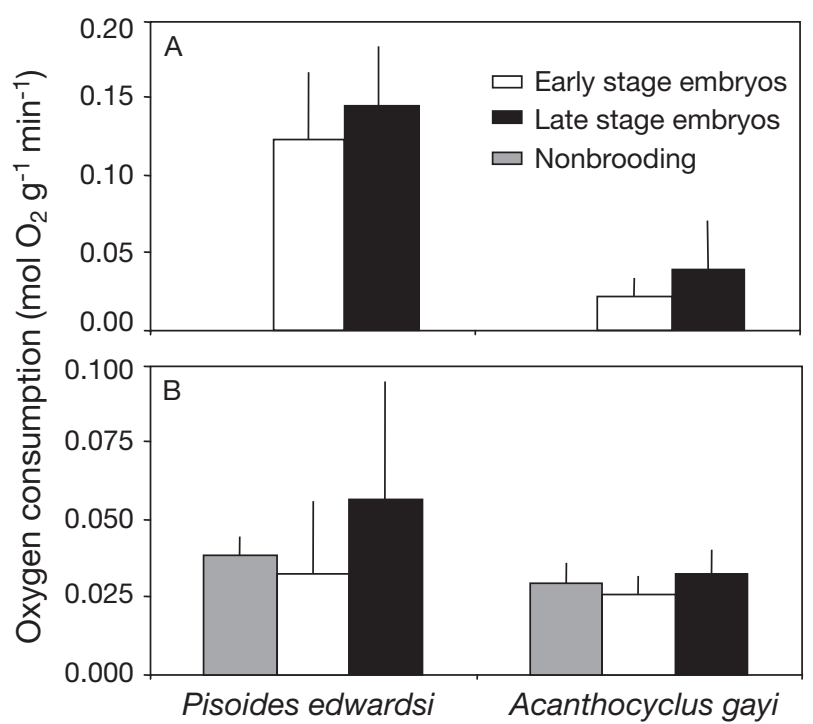

Fig. 2. Acanthocylus gayi and Pisoides edwardsi. (A) Mean oxygen consumption of embryos (per g wet weight) in early and late stage of development. (B) Mean oxygen consumption of females carrying early and late stage embryos and nonbrooding females (per g wet weight). Error bars are $+1 \mathrm{SD}$

on the behaviour that assesses oxygen condition in the embryo mass may occur at larger sizes. In contrast, the increase in the frequency of abdominal flapping is already evident between $P$. edwardsi and $A$. gayi, with a $65 \%$ increase in abdominal flapping frequency (Fig. 3B). Females of larger species carrying later stage embryos exhibited a frequency of abdominal flapping 2 to 5 times higher than A. gayi and P. edwardsi, respectively, at similar temperatures ( $M$. squinado: Fernández et al. 2000; C. setosus: Baeza \& Fernández 2002; H. plana: Fernández et al. 2003; O. trimaculatus: Fernández \& Brante 2003). This suggests that oxygen constraints occur already at the size of embryo masses exhibited by A. gayi, which triggers higher flapping frequency. It is remarkable that embryos of the 2 small body size species are always exposed to high oxygen conditions (>50\% air saturation), which contrasts with the low proportion of time that early stage embryos of large body size species are exposed to normoxia (Baeza \& Fernández 2002, Fernández et al. 2002, Brante \& Fernández 2003; Fig. 3C). We think that differences in flapping frequency throughout development between the small body size species, which do not seem to correspond directly to oxygen conditions in the embryo mass or oxygen demand of the embryos, can be explained by a combination of 3 factors: embryo mass size (correlated with female size), embryo size, and female behaviour. There is not enough information to carry out a similar comparison for the manipulation of the embryo mass using the pereiopods across body sizes.
The relationship between the frequency of abdominal flapping and female size can also be observed throughout embryo development. Abdominal flapping frequency increases as embryo development advances in larger species (Baeza \& Fernández 2002; Brante \& Fernández 2003), which seems to be related to embryo oxygen demand (Fernández et al. 2002). For instance, a $17 \%$ increase in oxygen consumption throughout development was reported for embryos of Ovalipes trimaculatus (70 to $80 \mathrm{~mm}$ carapace width), while the frequency of abdominal flapping of brooding females showed a 2-fold increase throughout embryo development (Fernández \& Brante 2003). The $100 \%$ increase in oxygen consumption during embryo development in Cancer setosus (100 to $130 \mathrm{~mm}$ carapace width), is associated with a 10-fold increase in abdominal flapping frequency (Baeza \& Fernández 2002). However, Acanthocyclus gayi showed a 7 -fold increase in the frequency of abdominal flapping, in spite of the fact that the $55 \%$ increase in oxygen consumption from early to late stage embryos was not significant. The $28 \%$ increase in mean oxygen consumption between early and late stage embryos of Pisoides edwardsi was not significant either, but in this case no change in the frequency of abdominal flapping during embryo development was observed. Similar (but significant) differences in oxygen consumption between early and late stage embryos have a tremendous impact on female behaviour in larger body size species (e.g. O. trimaculatus). Two unexpected results that deserve special attention are the enormous difference in embryo oxygen consumption of the 2 model species, and the lack of difference in oxygen consumption between early and late stage embryos. With respect to the patterns of embryo oxygen consumption, similar differences in embryo oxygen consumption have been reported for other crab species at similar temperatures (Baeza \& Fernández 2002, Brante et al. 2003, 2004). We attribute the lack of statistical differences in mean oxygen consumption between early and late stage embryos, which is a common pattern in nature (Naylor et al. 1999, Taylor \& Leelapiyanart 2001), to the variability in oxygen consumption of crab embryos. We think that the lack of difference in oxygen consumption between early and late stage embryos cannot be due to the broad categories of embryo stage because the same categories were used in other studies (Baeza \& Fernández 2002, Fernández et al. 2003, Brante et al. 2003).

The similarity in behavioural patterns in Pisoides edwardsi between females carrying early and late stage embryos deserves special consideration. It is the only species showing no change in behaviour throughout embryo development. We think that the combined effect of female behaviour, small body size, and large embryo size in $P$. edwardsi can explain the behavioural 


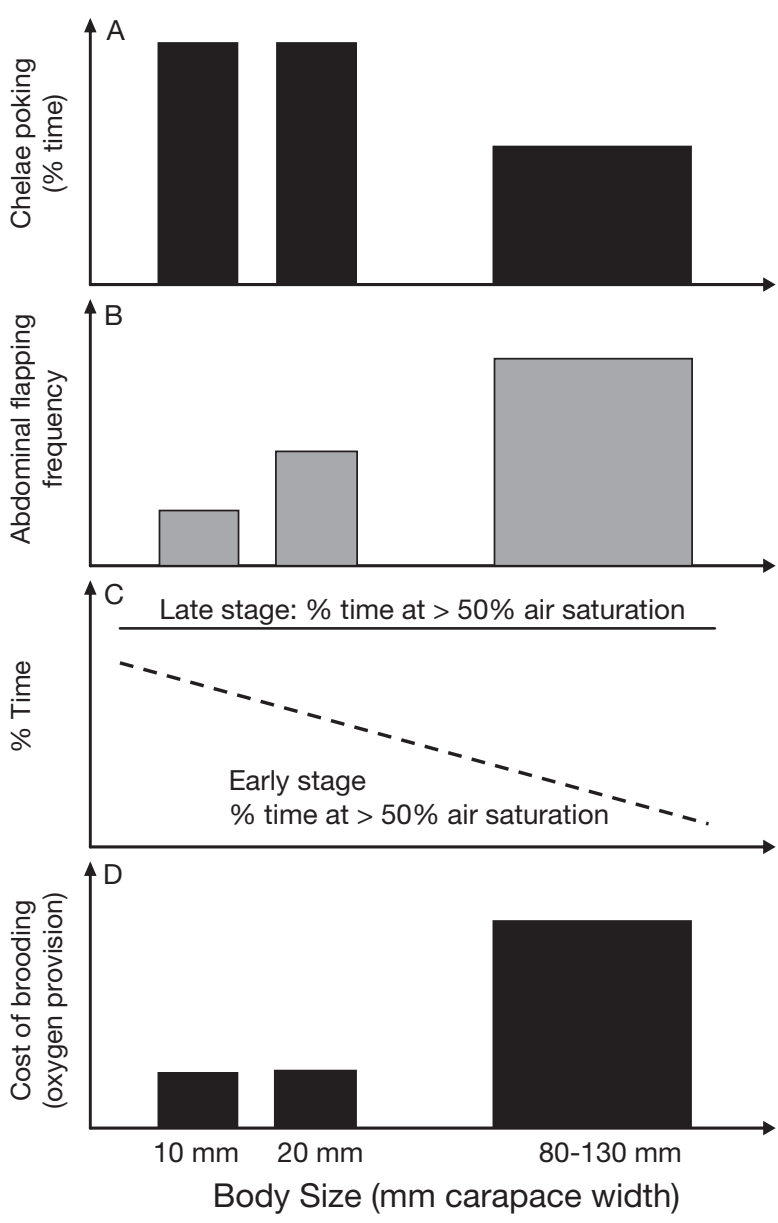

Fig. 3. Summary of the results of this study and existing published information on $(\mathrm{A}, \mathrm{B})$ brooding behaviour, $(\mathrm{C})$ on patterns of oxygen availability in the center of the embryo mass, in females carrying early and late stage embryos, and (D) on brooding costs in crab species at different body sizes. Brooding behavior was measured either as mean percent of time that females devoted to a particular behavior (e.g., chelae poking) or the number of events $\mathrm{h}^{-1}$ (e.g. abdominal flapping). Patterns of oxygen availability indicate the mean $\%$ of time that embryos were exposed to low $(<25 \%)$ and high $(>50 \%)$ levels of air saturation. Differences in oxygen consumption $\left(\mathrm{g}^{-1}\right.$ wet weight) between brooding and nonbrooding females carrying late stage embryos were used as a proxy of the cost of brooding. The comparison includes only reference to studies conducted at similar temperatures $\left(11\right.$ and $\left.12^{\circ} \mathrm{C}\right)$

pattern exhibited by this species. Certainly, a greater potential for a passive supply of oxygen (e.g. passive diffusion, interstitial flow of water) may occur in this small species. However, the role of female behaviour is still important, since passive oxygen supply alone cannot explain the oxygen conditions in the embryo mass of $P$. edwardsi. Gelatinous embryo masses, which largely depend on oxygen diffusion, show permanent hypoxia at similar embryo mass size (up to $8 \mathrm{~mm}$ diameter; Cohen \& Strathmann 1996). Embryo size also affects female brooding patterns (Taylor \& Leelapiyanart
2001). The embryo mass of $P$. edwardsi is entirely contained between the abdomen and the cephalothorax during early embryo development, but not during late embryo stages. We conclude that this change is due to the increase in embryo volume, which might facilitate passive supply of oxygen into the embryo masses because: (1) the embryo mass is less densely packed (Taylor \& Leelapiyanart 2001), and (2) the embryos may be exposed to water currents that supply oxygen, regardless of female abdominal flapping. Embryo volume increases $80 \%$ throughout development in P. edwardsi (pers. obs.).

Our results show that females of the 2 small species perform the same active brooding behaviour as large species. However, mean oxygen consumption of brooding females is not affected. These results contrast with previous studies on large species, showing a 2 to 3 -fold increase in oxygen consumption by females carrying later stage embryos when compared to females carrying early stage embryos, or nonbrooding females (i.e. Cancer setosus: Baeza \& Fernández 2002; Homalaspis plana: Ruiz-Tagle et al. 2002; Ovalipes trimaculatus: Fernández \& Brante 2003; Fig. 3D). This pattern suggests that the costs of oxygen provision may increase with body size, as brood weight is proportional to female weight (Hines 1982), constraining the capacity to brood at large body size. The costs of brooding may have consequences for other life history variables such as the number of broods per year, or female survival after reproduction (Brokordt et al. 2000, 2003). In fact, there is evidence showing a tradeoff between investment in eggs and the cost of providing oxygen to the embryos across latitude, as the cost of oxygen provision increases with temperature in brachyuran crabs (Brante et al. 2003). The increasing cost of brooding with body size in active brooding species is in line with previous evidence showing an allometric relationship between the increase in energy invested in facilitating oxygen diffusion (gel) and the size of gelatinous embryo masses in gastropods (Lee \& Strathmann, 1998). This study suggests that the cost of brooding increases with body size. Our results provide direct evidence for the effect of adult size on the costs of providing oxygen to the brood. Further, our study suggests that other factors, such as embryo size, play a critical role particularly for species with small body sizes (see also Taylor \& Leelapiyanart 2001). At large body size, embryo size seems to have lower impact on the cost of oxygen provision. The size of embryos of Maja squinado and Homalaspis plana were twice the size of the embryos of Cancer setosus and Ovalipes trimaculatus (Brante et al. 2004). However, the cost of brooding in these species did not show any relationship with embryo size (Fernández et al. 2000, Baeza \& Fernández 2002, 
Fernández et al. 2003, Fernández \& Brante 2003). We suggest, based on this study and evidence in the literature, that the high costs of brooding at large body size may be the mechanism behind the association between small body size and brooding reported for marine invertebrates (Strathmann \& Strathmann 1982).

Acknowledgements. We are grateful to K. Jeno for her help in the laboratory. We also thank F. Finke, J. Holl and 2 anonymous reviewers for their helpful comments on the manuscript. This study was fully funded by FONDECYT 1020860 (to M.F.). We also acknowledge the Humboldt Foundation for the instruments used for laboratory experiments. M. F. acknowledges the FONDAP-Fondecyt grant 1501-0001 to the Center for Advanced Studies in Ecology \& Biodiversity and the International Laboratory DIAMS.

\section{LITERATURE CITED}

Baeza JA, Fernández M (2002) Active brood care in Cancer setosus (Crustacea: Decapoda): the relationship between female behaviour, embryo oxygen consumption and the cost of brooding. Funct Ecol 16:241-251

Booth D (1995) Oxygen availability and embryonic development in sand snail (Polinices sordidus) egg masses. J Exp Biol 198:241-247

Brante A, Fernández M, Eckerle L, Marck F, Pörtner HO, Arntz W (2003) Reproductive investment in the crabs Cancer setosus along a latitudinal cline: egg production, embryo losses and embryo ventilation. Mar Ecol Prog Ser 251:221-232

Brante A, Cifuentes S, Pörtner HO, Arntz W, Fernández M (2004) Latitudinal comparisons in reproductive parameters in five brachyuran species along the Chilean coast. Rev Chil Hist Nat 77:15-27

Brokordt KB, Himmelman JH, Guderley H (2000) Effect of reproduction on escape responses and muscle metabolic capacities in the scallop Chlamys islandica (Müller 1776). J Exp Mar Biol Ecol 251:205-225

Brokordt KB, Guderley H, Guay M, Gaymer C, Himmelman JH (2003) Sex differences in reproductive investment: maternal care reduces escape response capacity in the whelk Buccinum undatum. J Exp Mar Biol Ecol 291:161-180

Cohen CS, Strathmann RR (1996) Embryos at the edge of tolerance: effects of environment and structure of egg masses on supply of oxygen to embryos. Biol Bull 190:8-15

Crump M (1996) Parental care among amphibians. Adv Study Behav 25:109-144

Fernández M, Brante A (2003) Brood care in brachyuran crabs: the effect of oxygen provision on reproductive costs. Rev Chil Hist Nat 76:157-168

Fernández M, Bock C, Pörtner HO (2000) The cost of being a caring mother: the ignored factor in the reproduction of marine invertebrates. Ecol Lett 3:487-494

Editorial responsibility: Otto Kinne (Editor-in-Chief), Oldendorf/Luhe, Germany
Fernández M, Pardo LM, Baeza JA (2002) Patterns of oxygen supply in embryo masses of brachyuran crabs throughout development: the effect of oxygen availability and chemical cues in determining female brooding behaviour. Mar Ecol Prog Ser 245:181-190

Fernández M, Ruiz-Tagle N, Cifuentes S, Pörtner HO, Arntz W (2003) Oxygen-dependent asynchrony of embryonic development in embryo masses of brachyuran crabs. Mar Biol 142:559-565

Green B, McCormick M (2005) Oxygen replenishment to fish nets: males adjust brood care to ambient conditions and brood development. Behav Ecol 16:389-397

Hines AH (1982) Allometric constraints and variables of reproductive effort in brachyuran crabs. Mar Biol 69: 309-320

Lardies MA, Fernández M (2002) Effect of oxygen availability in determining clutch size in Acanthina monodon. Mar Ecol Prog Ser 230:139-146

Lee C, Strathmann RR (1998) Scaling of gelatinous clutches: effects of sibling competition for oxygen on clutch size and parental investment per offspring. Am Nat 151:293-310

Naylor J, Taylor E, Bennett D (1999) Oxygen uptake of developing eggs of Cancer pagurus (Crustacea: Decapoda: Cancridae) and consequent behaviour of the ovigerous females. J Mar Biol Assoc UK 79:305-315

Ruiz-Tagle N, Fernández M, Pörtner HO (2002) Full time mothers: daily rhythms in brooding and nonbrooding behaviours of brachyuran crabs. J Exp Mar Biol Ecol 276:31-47

Seymour RS, Roberts DJ (1991) Embryonic respiration and oxygen distribution in foamy and non-foamy egg masses of the frog Limnodynastes tasmaniensis. Physiol Zool 64:1322-1340

Seymour RS, Geiser F, Bradford DF (1991) Gas conductance of the jelly capsule of terrestrial frog eggs correlates with embryonic stage, not metabolic demand or ambient $\mathrm{PO}_{2}$. Physiol Zool 68:206-222

Strathmann RR, Chaffee C (1984) Constraints on egg masses. II. Effect of spacing, size, and number of eggs on ventilation of masses of embryos in jelly, adherent groups, or thin-walled capsules. J Exp Mar Biol Ecol 84:85-93

Strathmann RR, Strathmann M (1982) The relationship between adult size and brooding in marine invertebrates. Am Nat 119:91-101

Strathmann RR, Strathmann M (1989) Evolutionary opportunities and constraints demonstrated by artificial gelatinous egg masses. In: Ryland JS, Tyler PA (eds) Proc 23rd Eur Mar Biol Symp, Swansea: Reproduction, genetics and distribution of marine organisms. Olsen \& Olsen, Fredensborg, p 201-209

Strathmann RR, Strathmann M (1995) Oxygen supply and limits on aggregation of embryos. J Mar Biol Assoc UK 75:413-428

Taylor HH, Leelapiyanart N (2001) Oxygen uptake by embryos and ovigerous females of 2 intertidal crabs, Heterozius rotundifrons (Belliidae) and Cyclograpsus lavauxi (Grapsidae): scaling and the metabolic costs of reproduction. J Exp Biol 204:1083-1097

Woods HA (1999) Egg-mass size and cell size: effects of temperature on oxygen distribution. Am Zool 39:244-252

Submitted: December 12, 2005; Accepted: February 9, 2006 Proofs received from author(s): March 3, 2006 